\title{
Core level photoelectron spectroscopy on the lanthanide-induced hydrolysis of DNA
}

\author{
Hidemi Shigekawa, ${ }^{a}$, b) Hiroyuki lkawa, and Ryozo Yoshizaki ${ }^{\mathrm{c})}$ \\ Center for Tsukuba Advanced Research Alliance, and Institute of Materials Science, University of Tsukuba, \\ Tsukuba 305, Japan \\ Yoshitoki lijima \\ Application and Research Center, JEOL Limited, Akishima, Tokyo 196, Center for Tsukuba Advanced \\ Research Alliance, University of Tsukuba, Tsukuba 305, Japan \\ Jun Sumaoka and Makoto Komiyama ${ }^{\text {a) }}$ \\ Department of Chemistry and Biotechnology, Graduate School of Engineering, University of Tokyo, Tokyo \\ 113, Center for Tsukuba Advanced Research Alliance, University of Tsukuba, Tsukuba 305, Japan
}

(Received 6 November 1995; accepted for publication 2 January 1996)

\begin{abstract}
The electronic structures of the complexes of diphenyl phosphate (DPP), a model compound of DNA, with lanthanide ions have been investigated to shed light on the mechanism of the cerium (IV)-induced nonenzymatic hydrolysis of DNA. Binding energies of the P $2 p$ core level of DPP were $134.2 \mathrm{eV}$ for the complexes with $\mathrm{La}(\mathrm{III}), \mathrm{Eu}(\mathrm{III})$, and $\mathrm{Lu}(\mathrm{III})$, and was $134.4 \mathrm{eV}$ for the $\mathrm{Ce}(\mathrm{IV})$ complex, when the metal/DPP molar ratio was $1: 1$. When the molar ratio was increased, only $\mathrm{Ce}(\mathrm{IV})$, the most active metal ion for DNA hydrolysis, showed a chemical shift of $\sim 0.5 \mathrm{eV}$ toward the higher binding energy region. The chemical shift of $\sim 0.5 \mathrm{eV}$ toward the higher binding energy region. The chemical shift was due to the systematic increase in the intensity of the higher binding energy component. The observed change in the electronic structure of the DPP-Ce(IV) complex may be related to the superb ability of Ce(IV) for the hydrolysis of DNA. (C) 1996 American Institute of Physics. [S0003-6951(96)03110-0]
\end{abstract}

Nonenzymatic scission of DNA has been attracting interest, because of potential application to molecular biology, biotechnology and therapy. ${ }^{1}$ However the phosphodiester linkages therein are enormously resistant to hydrolysis: the half-life of the linkages at $p \mathrm{H} 7$ and $25^{\circ} \mathrm{C}$ is estimated to be 200 million years. It was only in 1992 that the first nonenzymatic hydrolysis of linear DNAs was achieved by the authors by use of lanthanide ions. ${ }^{2-5}$ The cerium(IV) ion is the most active. ${ }^{6-8}$ It decreases the half-life to a few hours corresponding to an acceleration around $10^{11}$ fold. Quite interestingly, the remarkable catalysis is totally specific to lanthanide ions, especially to the Ce(IV) ion. In addition, an artificial nuclease, which selectively hydrolyzes the target phosphodiester linkage in DNA and thus is a useful tool for molecular biology, has been synthesized by the attachment of $\mathrm{Ce}$ (IV) ion (in the form of iminodiacetate complex) to a DNA oligomer. ${ }^{9}$ However, it has not yet been understood why the $\mathrm{Ce}(\mathrm{IV})$ ion overwhelmingly exceeds the other lanthanide ions more than 100-fold in the activity.

In order to understand the scission mechanism, information about the change in the electronic structure of the phosphodiester linkage of nucleic acids during complex formation with lanthanide ions, is urgently required. In this letter, we present the results of core level photoelectron spectroscopy ${ }^{10}$ on the complexes of diphenyl phosphate (DPP), a model sample of nucleic acids lanthanide. As shown in Fig. 1, DNA and DPP have similar phosphodiester linkages.

\footnotetext{
a) Authors to whom correspondence should be addressed.

b)Electronic mail: hidemi@mat.ims.tsukuba.ac.jp

${ }^{c}$ Permanent address: Institute of Applied Physics, and Cryogenics Center, University of Tsukuba, Tsukuba, Japan.
}

The specimens were prepared by mixing DPP with the corresponding metal salt $\left[\mathrm{Ce}\left(\mathrm{NH}_{4}\right)_{2}\left(\mathrm{NO}_{3}\right)_{6}, \mathrm{LaCl}_{3}, \mathrm{EuCl}_{3}\right.$, or $\left.\mathrm{LuCl}_{3}\right]$ in water at a required molar ratio. After the mixtures were centrifugalized, the sediment was collected and dried in vacuum, and was then directly subjected to XPS analysis. The electronic structure of the phosphodiester linkage in the DPP molecules in the presence of lanthanide ions was studied by measuring the $\mathrm{P} 2 p$ core level spectra. X-ray used for excitation was $\mathrm{Mg} K \alpha(h \nu=1253.6 \mathrm{eV})$, and energy resolution was $0.9 \mathrm{eV}$ at $\mathrm{Ag} 3 d_{5 / 2} .{ }^{11}$ The obtained binding energies were calibrated using the $\mathrm{CO}$ bonding state $(\mathrm{O} 1 \mathrm{~s}$, $533.2 \mathrm{eV})$ existing in all the samples. The measurements were performed in a vacuum of $5 \times 10^{-7} \mathrm{~Pa}$. Since absence of the hydrolysis of DPP during the spectroscopy has been confirmed by a reversed-phase high performance liquid chro-

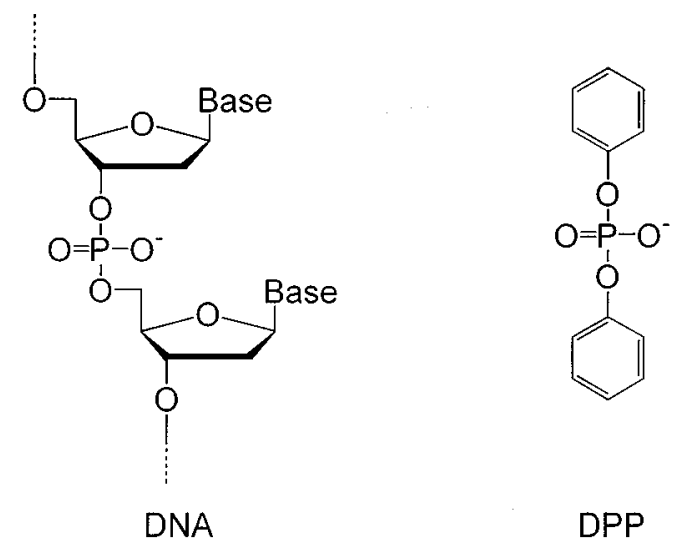

FIG. 1. Chemical structures of the phosphodiester linkage in DNA and DPP. 

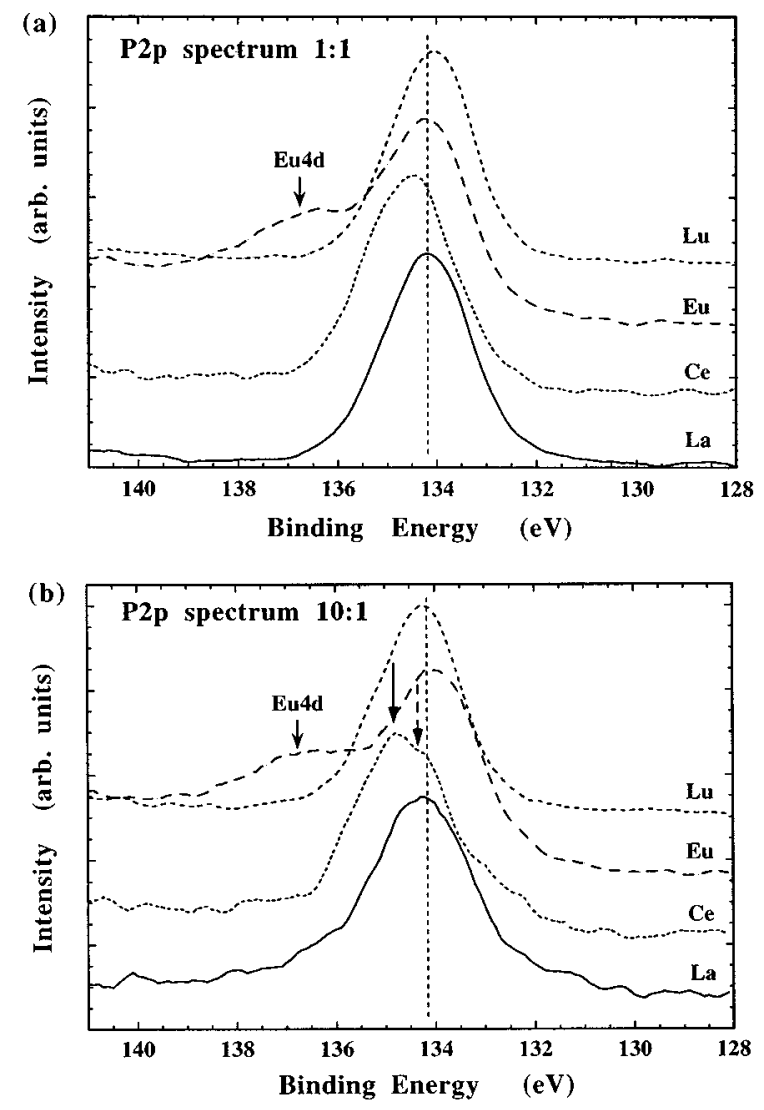

FIG. 2. Core level spectra of P $2 p$ obtained for the DPP complex with $\mathrm{La}(\mathrm{III}), \mathrm{Ce}(\mathrm{IV}), \mathrm{Eu}(\mathrm{III})$, and $\mathrm{Lu}(\mathrm{III})$ for the molar ratio of (a) $1: 1$ and (b) $10: 1$.

matography, the observed spectra are related to the electronic structures of the DPP-Ce(IV) complex at the stage immediately before the phosphodiester linkage is broken.

Figure 2 shows the core level spectra of $\mathrm{P} 2 p$ for the mixtures of lanthanide ions and DPP with a molar ratio of $1: 1$ and $10: 1{ }^{12}$ When the metal/DDP molar ratio was $1: 1$, the binding energy of the $\mathrm{P} 2 p$ core level was observed at 134.2 $\mathrm{eV}$ for the complexes with $\mathrm{La}(\mathrm{III}), \mathrm{Eu}(\mathrm{III})$, and $\mathrm{Lu}(\mathrm{III})$ ions. For the Ce(IV) complex, however, the $\mathrm{P} 2 p$ core level had a slightly higher binding energy of $134.4 \mathrm{eV}$. Quite significantly, only $\mathrm{Ce}(\mathrm{IV})$ induced a chemical shift of $\sim 0.5 \mathrm{eV}$ toward the higher binding energy region as indicated by the arrows in Fig. 2(b), when the molar ratio was increased from $1: 1$ to $10: 1$. The activity of Ce(IV) for the modulation of the electronic structure of the phosphodiester linkage is remarkably greater than those of other lanthanide ions. ${ }^{12}$ This is, to our best knowledge, the first spectroscopic evidence showing a clear-cut difference between Ce(IV) and other lanthanide ions in the interaction with phosphodierster linkages.

Figure 3 shows a series of $\mathrm{P} 2 p$ core level spectra for the samples of Ce(IV)/DPP molar ratios of (a) 0:1, (b) 1:1, (c) 2:1, (d) 5:1, and (e) 10:1. The spectra of Figs. 3(b)-3(e) could be decomposed into two spin doublet components $P_{H}$ and $P_{L}$, which have higher and lower binding energies, respectively. When the molar ratio was $1: 1$, the main component was the peak $P_{L}$ as shown in Fig. 3(b). However, with the increase of the molar ratio, the intensity of peak $P_{H}$ increased [Figs. 3(c)-3(e)]. The change in the relative

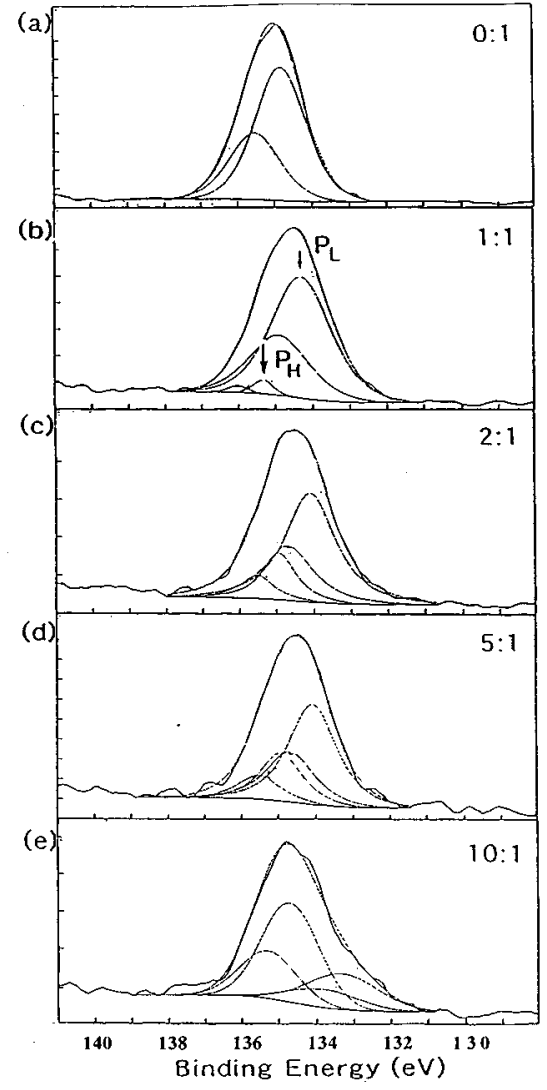

FIG. 3. Core level spectra of P $2 p$ observed for the Ce(IV)/DPP mixtures, with the molar ratios of (a) 0:1, (b) 1:1, (c) 2:1, (d) 5:1, and (e) 10:1. $P_{H}$ and $P_{L}$ correspond to the doublet peaks of higher and lower binding energies.

intensity of the two components in Fig. 3 is quantitatively presented in Fig. 4. A possible explanation for the observed P $2 p$ core level spectra is that, component $P_{L}$ corresponds to the bonding of the phosphodiester linkage with $\mathrm{Ce}(\mathrm{IV})$ ion, and the component $P_{H}$ corresponds to the simultaneous bonding with two $\mathrm{Ce}(\mathrm{IV})$ ions. The full width at halfmaximum (FWHM) increased with increasing molar ratio; from 0.31 to $0.90 \mathrm{eV}$ for peak $P_{H}$, and 0.93 to $1.08 \mathrm{eV}$ for peak $P_{L}$ [Figs. 3(b)-3(e)]. From the observed chemical shift and the change in the FWHM of the two components, the chemical reactivity of the phosphodiester linkage in a DPP

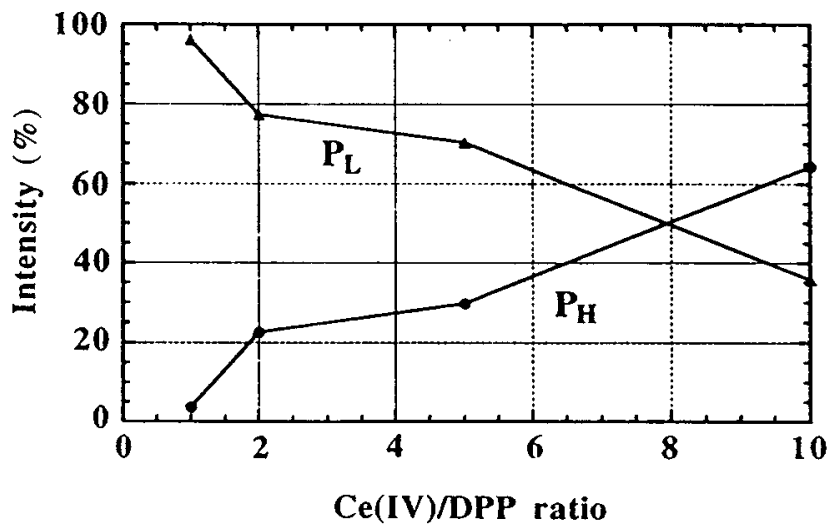

FIG. 4. Change in the relative intensity of the two $\mathrm{P} 2 p$ components $P_{H}$ and $P_{L}$ in Fig. 3. 


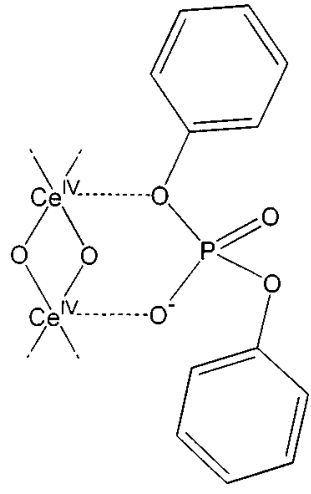

FIG. 5. Schematic model for the structure of the 2:1 complex between Ce(IV) ion and DPP.

molecule seems to be increased, on the complex formation with two $\mathrm{Ce}(\mathrm{IV})$ ions.

Proposed structure of the 2:1 complex between Ce(IV) ion and DPP is schematically depicted in Fig. 5. In addition to the usual coordination of a Ce(IV) ion to the phosphate, another $\mathrm{Ce}(\mathrm{IV})$ ion in the hydroxide cluster interacts with one of the other three oxygen atoms around the phosphorus atom (the interaction with the phenoxide oxygen atom is presented in the figure). The mutual orientation of the two $\mathrm{Ce}(\mathrm{IV})$ ions in the hydroxide cluster should be favorable for the multiple interactions. Because of the significant electron withdrawal by the cooperation of the two $\mathrm{Ce}$ (IV) ions, the electron density on the phosphorus atom is greatly decreased, exactly as observed in the present photoelectron spectroscopy (the peaks $P_{H}$ in Fig. 3). This effect renders the highly stable phosphodiester linkage susceptible to hydrolysis under mild conditions. The activation of the linkage by the complex formation with only one metal ion (corresponding to the peak $P_{L}$ in Fig. 3) is insufficient. The DNA hydrolysis proceeds via nucleophilic attack by the $\mathrm{Ce}(\mathrm{IV})$-bound hydroxide toward the phosphorus atom. ${ }^{8}$ The superiority of Ce(IV) over other lanthanide ions and nonlanthanide ions is presumably ascribed to a better orientation of two metal ions in the hydroxide cluster and/or larger acidity, which makes the synergetic catalysis much more effective. According to a recent $a b$ initio calculation, bonding of a single Ce(IV) atom to DPP does not result in breaking of the phosphodiester linkage. ${ }^{13}$ Further information on the chemical structure of the Ce(IV) complexes is necessary to clarify the details.

The spectroscopic evidence is totally consistent with the following kinetic results showing that the rate of the $\mathrm{Ce}(\mathrm{IV})$ induced DNA hydrolysis monotonously decreases with decrease in the molar ratio of the $\mathrm{Ce}$ (IV) ion to the substrate DNA. The rate constants of the hydrolysis of thymidylyl $\left(3^{\prime}-5^{\prime}\right)$ thymidine at $\mathrm{pH} 7$ and $50{ }^{\circ} \mathrm{C}$ are 0.33 , 0.03 , and $0.00 \mathrm{~h}^{-1}$, respectively, when the $\mathrm{Ce}(\mathrm{IV}) /$ substrate ratios are 100,10 , and 1 . Apparently, two or more $\mathrm{Ce}(\mathrm{IV})$ ions, with respect to the phosphodiester linkage, are required for the efficient DNA hydrolysis.

Finally, it is noteworthy that the $\mathrm{Zr}(\mathrm{IV})$ ion, which is not active for DNA hydrolysis, caused virtually no change to the $\mathrm{P} 2 p$ binding energy of DPP at the metal/DPP ratio 10 (data not presented). Thus, the significant change by the Ce(IV) ion is never simply ascribed to its large positive charge. It is strongly indicated that some specific interactions between the orbital(s) of the Ce(IV) ion and those of DPP is responsible for the change (and also presumably for the remarkable catalytic activity of the $\mathrm{Ce}$ (IV) ion for DNA hydrolysis).

In conclusion, the binding energies of the $\mathrm{P} 2 p$ core level were rather similar for the DPP mixtures with La, $\mathrm{Ce}(\mathrm{IV}), \mathrm{Eu}$, and Lu, when the metal/DPP molar ratio was $1: 1$. However, when the molar ratio was increased, only $\mathrm{Ce}(\mathrm{IV})$ induced an apparent change in the spectra. The P $2 p$ core level spectra of the Ce(IV) complex were deconvoluted into two components, the intensity ratio and FWHM of which were found to change systematically with the increase in the Ce(IV)/DPP molar ratio. Since the DPP complex has a chemical structure similar to that of the DNA complex, the characteristic P $2 p$ core level spectra, observed only with $\mathrm{Ce}(\mathrm{IV})$, is expected to be related to the scission mechanism of DNA.

This work was supported in part by Grants-in-Aid for Scientific Research on Priority Areas (New Development of Rare-Earth Complexes), from the Ministry of Education, Science and Culture of Japan. Support from Shigekawa project of TARA, University of Tsukuba is also acknowledged.

${ }^{1}$ P. B. Dervan, Science 232, 464 (1986).

${ }^{2}$ Y. Matsumoto and M. Komiyama, Nucl. Acids Symp. Ser. 27, 33 (1992).

${ }^{3}$ M. Komiyama, K. Matsumura, K. Yonezawa, and Y. Matsumoto, Chem. Express 8, 85 (1993).

${ }^{4}$ T. Shiiba, K. Yonezawa, N. Takeda, Y. Matsumoto, M. Yashiro, and M. Komiyama, J. Mol. Catal. 84, L21 (1993).

${ }^{5}$ M. Komiyama, Y. Matsumoto, T. Shiiba, N. Hayashi, and K. Watanabe, Polymer J. 25, 1211 (1993).

${ }^{6}$ M. Komiyama, T. Kodama, N. Takeda, J. Sumaoka, T. Shiiba, Y. Matsumoto, and M. Yashiro, J. Biochem. 115, 809 (1994).

${ }^{7}$ B. Takasaki and J. Chin, J. Am. Chem. Soc. 116, 1121 (1994).

${ }^{8}$ M. Komiyama, N. Takeda, Y. Takahashi, H. Uchida, T. Shiiba, T. Kodama, and M. Yashiro, J. Chem. Soc. Perkin Trans. 2, 269 (1995); and references therein.

${ }^{9}$ M. Komiyama, T. Shiiba, H. Uchida, Y. Takahashi, N. Takeda, K. Matsumura, and T. Kodama, Supramol. Chem. 4, 31 (1994).

${ }^{10}$ Y. Ma, C. T. Chen, G. Meigs, G. Illing, and H. Shigekawa, Phys. Rev. B 45, 5961 (1992).

${ }^{11}$ Y. Iijima, K. Muramoto, M. Uemura, and K. Hirooka, Jpn. J. Appl. Phys. 32, 5080 (1993)

${ }^{12}$ The chemical shift change due to the $1: 1$ complex formation could not be estimated here, since the spectrum for the free anion of DPP is not available at present. The specimen for the free DPP used here was prepared from acidic solutions, and DPP therein is protonated. Thus, we focus the present analysis to the comparison of the spectral change of DPP on the complex formation with the Ce(IV) ion with the corresponding values for other lanthanide ions.

${ }^{13}$ K. Hirao (private communication). 